\title{
Joint FAO/IAEA Coordinated Research Project on "Exploring genetic, molecular, mechanical and behavioural methods of sex separation in mosquitoes" - an introduction
}

\author{
Kostas Bourtzis ${ }^{1 *}$ and Zhijian Jake Tu
}

Aedes and Anopheles mosquitoes are major vectors of human diseases such as malaria, dengue, chikungunya, Zika and yellow fever. There is a lack of efficient drugs and vaccine for most of these vector-borne infectious diseases and traditional mosquito control methods, mainly based on larval source reduction and insecticides are inefficient. Therefore, new or complementary techniques, such as novel genetic strategies, are urgently needed to combat major mosquito vector species. There are three major types of genetic strategies to achieve vector population suppression: the sterile insect technique (SIT), the incompatible insect technique (IIT), and transgenic approaches. These techniques all rely on male-only releases as female mosquitoes bite and transmit pathogens. However, the lack of efficient and robust sex separation methods presents a major bottleneck to the successful application of these techniques as the currently available sex separation methods are inadequate for safe and bio-secure male-only releases in large scale operational programs $[1,2]$.

Five years ago, a coordinated research project (CRP) entitled "Exploring genetic molecular, mechanical and behavioural methods of sex separation in mosquitoes" was initiated under the auspices of the Joint Division of Nuclear Techniques in Food and Agriculture of the Food and Agriculture Organization (FAO) and the International Atomic Energy Agency (IAEA). Kostas Bourtzis, a member of the Insect Pest Control Subprogramme of the FAO/ IAEA, coordinated the project which included 19 scientists with a broad range of expertise from 14 countries, to

\footnotetext{
* Correspondence: K.Bourtzis@iaea.org

${ }^{1}$ Insect Pest Control Laboratory, Joint FAO/IAEA Programme of Nuclear

Techniques in Food and Agriculture, Vienna, Austria

Full list of author information is available at the end of the article
}

explore classical genetic, molecular, mechanical, behavioural, developmental and symbiont-based approaches for sex separation in mosquitoes. In the frame of this 5-year CRP project, four meetings were organized during which the participating scientists presented their findings and achievements as well as critically discussed and coordinated their research. This special issue presents, in the form of original and/or review papers, recent achievements in the field of mosquito sex separation, together with a perspective paper which discusses potential future steps.

Several review articles present the progress and challenges for the development of genetic sexing strains in Aedes and Anopheles mosquitoes by classical genetics and/or molecular-based approaches. Bernardini et al. [3] review the significant progress achieved during the last few years towards the development of genetic sexing strains using transgenic technologies for the control of Anopheles gambiae, which is a major vector of human malaria. Mashatola et al. [4] discuss various sex separation strategies that have been or are currently explored to develop a robust sex separation method which would allow SIT applications against $A n$. arabiensis including the exploitation of sex-specific behavioural and developmental differences as well as genetic-based approaches such as genetic sexing strains. Haecker \& Schetelig [5] present the pros and cons of using transposable elements, site-specific recombination, and genome editing methods in the context of developing genetic sexing strains for mosquito vector control. Araujo et al. [6] report on the need to deeply understand the mechanisms of sex determination in Aedes mosquitoes, which would allow the exploitation of the sex determination pathways to construct male-only strains. 
Ndo et al. [7] report the isolation, establishment and the characterization of a temperature-sensitive lethal (tsl) mutant strain in $A n$. arabiensis and describe its potential use for the development of a genetic sexing strain (GSS) similar to the one currently used worldwide for SIT applications against the major agricultural pest, the Mediterranean fruit fly Ceratitis capitata [8, 9]. Krzywinska \& Krzywinski [10] show that the ectopic expression of the sex determining gene Yob largely results in female lethality or sterility while males remain viable and fertile. These data suggest that it is possible to establish An. gambiae transgenic genetic sexing strains with a conditional male-only phenotype. Smidler et al. [11] investigate the promoter of the major mating plug protein Plugin and use it to express fluorescence markers in An. gambiae. Successful insemination by males of these transgenic lines can be detected up to eight hours after copulation by monitoring the deposition of the florescent markers in mated females, thus facilitating the assessment of male mating competitiveness. Using RNAseq data, Wu et al. [12] identify pure early zygotic genes (pEZGs) whose promoter or regulatory sequences could be used to drive female-specific lethality to achieve sex separation and male-only releases.

As regards Aedes species, Gomulski et al. [13] identify the male-specific chromosome 1 of Ae. albopictus and characterize the putative male-determining gene Nix which paves the way for unravelling the sex determination pathway in this species as well as the development of novel genetic control strategies against this mosquito vector species. In addition, $\mathrm{Hu} \& \mathrm{Tu}[14]$ provide evidence that the promoter of the endogenous early zygotic gene KLC2 is active at the syncytial blastoderm and early cellular blastoderm as well as in male testes suggesting that it could be harnessed for the development of genetic sexing strategies in Aedes aegypti.

Bellini et al. [15] report that, although the selection for enhanced dimorphism in pupal size is challenging in Aedes albopictus, it is possible to select strains for enhanced protandry which may result to robust and efficient sex separation approaches is support of sterile insect technique applications. Interestingly, Zacarés et al. [16] report on the development of an automated pupal size estimator which was used to show that enhanced sexual size dimorphism (SSD)-based sex sorting methods can result to highly efficient sex separation, and at the same satisfactory male pupae recovery, in Aedes aegypti, Ae. albopictus and Ae. polynesiensis. A significant breakthrough is presented by Lebon et al. [17] who constructed the first genetic sexing strain (GSS) in Ae. albopictus, namely Tikok, based on the $r d l$ gene conferring dieldrin resistance. Even though this GSS is based on an insecticide resistance gene, this achievement opens the door for large-scale sex separation critical for population suppression applications.
Using laboratory data and simulations, Moretti et al. [18] suggest that it is possible, under certain conditions, to apply in the field a Wolbachia bidirectional cytoplasmic incompatibility-based suppression strategy which will rely on the release of incompatible males together with a small percentage of contaminant females. However, the risk of such release strategies to end up in population replacement instead of population suppression would still exist $[19,20]$. In the absence of efficient and robust methods for sex separation, and to avoid the release of even a small number of fertile and potentially pathogen transmitting females, the combination of sterile insect technique and incompatible insect technique (SIT/IIT), which combines irradiation with Wolbachia symbiosis, was suggested [19, 20]. This integrated approach was first developed in Aedes albopictus and tested under laboratory conditions [21-23].

In this special issue, Kittayapong et al. [24] discuss that, during a small scale Ae. aegypti suppression trial of combined SIT/IIT approach in Thailand, and due to the inefficiency of the mechanical approach based on glass larval-pupal separators, there was an accidental release of a small number of females. However, the released females were completely sterile due to the integration of irradiation, so there was no genetic footprint in the environment. In addition, due to the presence of Wolbachia and their short life span, the irradiated Wolbachia-infected females are unable to transmit any pathogens.

As an epilogue in this special issue, Papathanos et al. [25] highlight in a perspective manuscript the remaining bottlenecks and challenges facing the development of suitable sex separation approaches for the deployment of mosquito genetic population control strategies. They also suggest future directions for the development of efficient and robust sex separation methods, ideally based on genetic sexing strains, for Anopheles and Aedes mosquitoes.

It is our hope, as guest editors, that this issue of the Parasites \& Vectors presents the current knowledge in the field of mosquito sex separation. We also hope that it will help to the design and support of future research activities for the development of efficient, robust and cost-effective sex separation methods which are urgently needed for large scale deployment of mosquito population control strategies.

\section{Acknowledgements}

Not applicable.

\section{Funding}

Publication costs for this study was provided by the International Atomic Energy Agency as part of the Coordinated Research Project "Exploring genetic, molecular, mechanical and behavioural methods of sex separation in mosquitoes".

Availability of data and materials Not applicable. 


\section{About this supplement}

This article has been published as part of Parasites \& Vectors Volume 11 Supplement 2, 2018: Exploring genetic molecular, mechanical and behavioural methods of sex separation in mosquitoes. The full contents of the supplement are available online at https://parasitesandvectors.biomedcentral.com/ articles/supplements/volume-11-supplement-2.

\section{Authors' contributions}

$\mathrm{KB}$ and ZJT wrote the manuscript. Both authors read and approved the final manuscript.

\section{Ethics approval and consent to participate}

Not applicable.

\section{Consent for publication}

Not applicable.

\section{Competing interests}

The authors declare that they have no competing interests.

\section{Publisher's Note}

Springer Nature remains neutral with regard to jurisdictional claims in published maps and institutional affiliations.

\section{Author details}

'Insect Pest Control Laboratory, Joint FAO/IAEA Programme of Nuclear Techniques in Food and Agriculture, Vienna, Austria. ${ }^{2}$ Department of Biochemistry, Virginia Tech, Blacksburg, VA 24061, USA.

Published: 24 December 2018

\section{References}

1. Papathanos PA, Bossin HC, Benedict MQ, Catteruccia F, Malcolm CA, Alphey $L$, Crisanti A. Sex separation strategies: past experience and new approaches. Malar J. 2009;8(Suppl. 2):S5.

2. Gilles J, Schetelig M, Scolari F, Marec F, Capurro ML, Franz G, Bourtzis K. Towards mosquito Sterile Insect Technique programmes: exploring genetic, molecular, mechanical and behavioural methods of sex separation in mosquitoes. Acta Trop. 2014;132(Suppl):178-87.

3. Bernardini F, Haghighat-Khah RE, Galizi R, Hammond AM, Nolan T, Crisanti A. Molecular tools and genetic markers for the generation of transgenic sexing strains in Anopheline mosquitoes. Parasit Vectors. 2018; (In press, this issue).

4. Mashatola T, Ndo C, Koekemoer LL, Dandalo LC, Wood OR, Malakoane L, et al. Sex separation tools for sterile insect technique applications to suppress populations of the malaria vector, Anopheles arabiensis: Progress, challenges and lessons learnt. Parasit Vectors. 2018; (In press, this issue).

5. Häcker I, Schetelig MF. Molecular tools to create sexing strains in mosquitoes. Parasit Vectors. 2018; (In press, this issue).

6. Araújo HRC, Kojin BB, Capurro ML. Sex determination and Aedes population control. Parasit Vectors. 2018; (In press, this issue).

7. Ndo C, Poumachu Y, Metitsi D, Awonoambenei HF, Tchuinkam T, JLR G, Bourtzis K. Isolation and characterization of a temperature-sensitive lethal strain of Anopheles arabiensis for SIT-based applications. Parasit Vectors. 2018; (In press, this issue).

8. Franz G. Genetic sexing strains in Mediterranean fruit fly, an example for other species amenable to large-scale rearing as required for the sterile insect technique. In: Dyck VA, Robinson AS, Hendrichs J, editors. Sterile Insect Technique. Principles and Practice in Area-Wide Integrated Pest Management. Dordrecht: Springer; 2005. p. 427-51.

9. Augustinos AA, Targovska A, Cancio-Martinez E, Schorn E, Franz G, Caceres C, et al. Ceratitis capitata genetic sexing strains: laboratory evaluation of strains from mass rearing facilities worldwide. Entomol Exp Appl. 2017;164:305-17.

10. Krzywinska I, Krzywinski J. Effects of stable ectopic expression of the primary sex determination gene Yob in the mosquito Anopheles gambiae. Parasit Vectors. 2018; (In press, this issue).

11. Smidler A, Scott S, Mameli E, Shaw WR, Catteruccia F. A transgenic tool to assess Anopheles mating competitiveness in the field. Parasit Vectors. 2018 (In press, this issue).

12. Wu Y, Hu W, Bielder J, Chen XG, Tu Z. Pure early zygotic genes in the Asian malaria mosquito Anopheles stephensi. Parasit Vectors. 2018; (In press, this issue).
13. Gomulski LM, Mariconti M, Di Cosimo A, Scolari F, Manni M, Savini G, et al. The Nix locus on the male-specific homologue of chromosome 1 in Aedes albopictus is a strong candidate for a male-determining factor. Parasit Vectors. 2018; (In press, this issue).

14. Wanqi Hu W, Zhijian TZ. Transgenic analysis identifies a functional early zygotic promoter and reveals that an early zygotic gene is also expressed in the testis in Aedes aegypti. Parasit Vectors. 2018; (In press, this issue).

15. Bellini R, Puggioli A, Balestrino F, Carrieri M, Urbanelli S. Exploring protandry and pupal size selection for Aedes albopictus sex separation. Parasit Vectors. 2018; (In press, this issue).

16. Zacarés M, Salvador-Herranz G, Almenar D, Tur C, Argilés R, Bourtzis K, et al. Exploring the potential of size distribution analysis for the sex sorting of different vector mosquito species. Parasit Vectors. 2018; (In press, this issue).

17. Lebon C, Benlali A, Atyame C, Mavingui P, Tortosa P. Construction of a genetic sexing strain for Aedes albopictus: a promising tool for the development of sterilizing insect control strategies targeting the tiger mosquito. Parasit Vectors. 2018; (In press, this issue).

18. Moretti R, Marzo GA, Lampazzi E, Calvitti M. Cytoplasmic incompatibility management to support a Wolbachia-based sterile insect technique against Aedes albopictus. Parasit Vectors. 2018; (In press, this issue).

19. Lees RS, Gilles JRL, Hendrichs J, Vreysen MJB, Bourtzis K. Back to the future: the Sterile Insect Technique against mosquito disease vectors. Curr Opin Insect Sci. 2015;10:156-62.

20. Bourtzis $K$, Lees RS, Hendrichs J, Vreysen MJB. More than one rabbit out of the hat: radiation, transgenic and symbiont-based approaches for sustainable management of mosquito and tsetse fly populations. Acta Trop. 2016;157:115-30.

21. Zhang D, Zheng X, Xi Z, Bourtzis K, Gilles JRL. Combining Sterile Insect Technique with Incompatible Insect Technique: I-impact of Wolbachia infection on the fitness of triple and double-infected strains of Aedes albopictus. PLoS One. 2015;10:e0121126.

22. Zhang $D$, Lees RS, Xi Z, Gilles JRL, Bourtzis K. Combining the Sterile Insect Technique with Wolbachia-based approaches: II-A safer approach to Aedes albopictus population suppression programmes, designed to minimize the consequences of inadvertent female release. PLoS One. 2015;10:e0135194.

23. Zhang D, Lees RS, Xi Z, Bourtzis K, Gilles JRL. Combining the sterile Insect Technique with the Incompatible Insect Technique: II-Robust mating competitiveness of irradiated triple Wolbachia-infected Aedes albopictus males under semi-field conditions. PLoS One. 2016;11:e0151864.

24. Kittayapong P, Kaeothaisong N, Ninphanomchai S, Limohpasmanee W. Combined sterile insect technique and incompatibility insect techniques: sex separation and quality of sterile Aedes aegypti male mosquitoes released in a pilot population suppression trial in Thailand. Parasit Vectors. 2018; (In press, this issue).

25. Papathanos PA, Bourtzis K, Tripet F, Bossin H, Jair Virginio JF, Capurro ML, et al. A perspective on the need and current status of efficient sex separation methods for mosquito genetic control. Parasit Vectors. 2018; (In press, this issue).

Ready to submit your research? Choose BMC and benefit from:

- fast, convenient online submission

- thorough peer review by experienced researchers in your field

- rapid publication on acceptance

- support for research data, including large and complex data types

- gold Open Access which fosters wider collaboration and increased citations

- maximum visibility for your research: over $100 \mathrm{M}$ website views per year

At $\mathrm{BMC}$, research is always in progress.

Learn more biomedcentral.com/submission 